\title{
REVISIONES
}

\section{Autodescubrimiento ético y deliberación: Hacia un modelo de enseñanza de la ética en el modelo por competencias}

\author{
Ethical self-discovery and deliberation: Towards a teaching \\ model of ethics under the competences model \\ Auto-descoberta ética e deliberação: Para um modelo de ensino \\ da ética no modelo das competências \\ Gómez, Verónica ${ }^{a}$; Royo, Paulina ${ }^{b}$

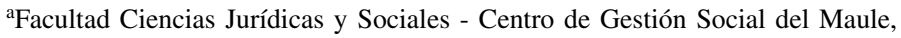 \\ Universidad Autónoma de Chile, Talca, Chile. Teléfono: 56-71-77666247. \\ Correo electrónico: vgomezu@uautonoma.cl \\ ${ }^{\mathrm{b}}$ Instituto de Estudios Humanísticos Juan Ignacio Molina, \\ Universidad de Talca, Talca, Chile. Teléfono: 56-71-2200485. \\ Correo electrónico: proyo@utalca.cl
}

\begin{abstract}
RESUMEN
Este artículo analiza una experiencia de enseñanza de la ética que enfatiza, particularmente, el desarrollo de las competencias dialógicas como eje clave de la reflexión en este ámbito, en una institución de educación superior de Talca, Chile. Partiendo del modelo de las éticas dialógicas y procedimentales, hemos implementado una metodología de enseñanza-aprendizaje orientada al autodescubrimiento ético y la construcción de acuerdos con otros. En este proceso, negociar valores con otras personas y ejercitar la empatía se han revelado como dos competencias clave, aunque poco desarrolladas, como muestran los resultados de la evaluación de esta experiencia. Ello, argumentamos, plantea un desafío importante para la educación cívica y el fortalecimiento de la democracia.
\end{abstract}

Palabras clave: enseñanza de la ética, juventud, modelo educativo por competencias, resolución de dilemas éticos, método deliberativo.

\begin{abstract}
This article analyzes a teaching experience in the domain of ethics under the competences model in a higher education institution in Talca, Chile. This experience focuses on the abilities required by civic dialogue as a key element for ethical reflection. Taking the dialogical- procedural model of ethics as a base, we propose a teaching methodology that emphasizes students' ethical self-discovery and the goal of reaching agreement by consensus. According to our data, two abilities are crucial for these processes: to negotiate values with others and to display empathy. Both, however, seem to be underdeveloped. We argue that this finding poses an important challenge to both civic education and the strengthening of democracy.
\end{abstract}

Key words: teaching and learning of ethics, young people, education by competences, resolution of ethical dilemmas, deliberative method.

\section{RESUMO}

Analisa-se uma experiência de ensino da ética que enfatiza, particularmente, o desenvolvimento das competências dialógicas como ponto-chave da reflexão nesse âmbito, em uma instituição de educação superior em Talca, Chile. Partindo do modelo das éticas dialógicas e procedimentais, tem-se implementado uma metodologia de ensino-aprendizagem orientada para a autodescoberta ética e para a construção de acordos com outras pessoas. Nesse processo, negociar valores com os demais e exercitar a empatia tem-se revelado como duas competências 
da maior importância, porém pouco desenvolvidas, segundo resultados da experiência. Isto revela um desafio fundamental para a educação cívica e o fortalecimento da democracia, argumenta-se.

Palavras chave: ensino da ética, juventude, modelo educativo por competências, resolução de dilemas éticos, método deliberativo.

\section{INTRODUCCIÓN}

Las nuevas generaciones enfrentarán un mundo cuya velocidad de transformación no tiene precedentes; un mundo más incierto e inseguro que el de décadas anteriores, producto de importantes transformaciones económicas, tecnológicas, políticas y culturales que han modificado la forma en que se organiza la sociedad. Uno de los cambios más importantes es el que ha ocurrido a nivel ideológico-valórico, desde el punto de vista de la pérdida de relevancia de los que fueran, tradicionalmente, los grandes sistemas de sentido: la religión, la tradición y las grandes ideologías políticas. Estos cambios influyen en la forma en que las personas conducen sus vidas, dependiendo cada vez menos de instituciones o referentes tradicionales y cada vez más de sus propias capacidades para interpretar la realidad y situarse en ella desde una visión de mundo y una jerarquía de valores que, de manera creciente, debe ser construida con recursos personales (PNUD, 2012).

Según la VII Encuesta Nacional de la Juventud, en la definición que hacen las personas jóvenes (definidas como el tramo etario que va de los 15 a los 29 años) de sí mismas, estas reconocen una preferencia por vivir en un ambiente seguro y evitar peligros (57\%), seguido por el cuidado del medioambiente (54\%), ser creativo y "hacer las cosas a su modo" (48\%) y hacer algo bueno para la sociedad (47\%). Un 44,6\% declaró considerar importante la tradición; seguir las costumbres que le han entregado su religión o familia, mientras que opciones como ser reconocido por tomar riesgos y ser rico se encuentran en puestos secundarios: $31 \%$ y 16\%, respectivamente (INJUV, 2012). La VII Encuesta Nacional de la Juventud también indica que los jóvenes chilenos tienen posturas diversas frente a la llamada "agenda valórica", y que el examen de los datos de sondeos anteriores revela que la identificación con los sectores políticos tradicionales -izquierda, centro, derecha- ha disminuido en los últimos años, al mismo tiempo que existe un incremento de quienes no se identifican con ningún sector o tendencia. De ese modo, el proyecto colectivo -el rol del Estado, el modelo económico y el lugar de las libertades individuales- es mirado con una cierta ambigüedad y desconfianza (INJUV, 2012).

De manera similar, el Informe de Desarrollo Humano en Chile de 2012 (PNUD, 2012) señala que el nivel de felicidad que declaran los individuos está relacionado con las condiciones sociales en las que se insertan las personas: lo que piensan y sienten está influenciado por el peso de las experiencias y contextos en los que se desenvuelven, por lo que las habilidades que las personas tengan para desarrollar sus proyectos de vida resultan clave. En este contexto de proyectos de vida múltiples y potencialmente divergentes, la capacidad de diálogo valórico y tolerancia es fundamental.

Este artículo presenta una experiencia educativa concreta orientada, justamente, hacia el fortalecimiento de las capacidades dialógicas (desde el punto de vista del diálogo cívico) de jóvenes universitarios de la ciudad de Talca, Chile. Nos detendremos, específicamente, en una metodología de enseñanza-aprendizaje basada en el modelo teórico de las éticas 
dialógico-procedimentales que parten, precisamente, de la necesidad de contar con una esfera pública vigorosa como condición indispensable para las sociedades contemporáneas. Una esfera pública que requiere, por cierto, de ciudadanos/as capaces de enfrentar los desafíos de la convivencia colectiva desde una doble perspectiva: a) la de explicitar sus propios proyectos de vida y sus implicaciones ético-morales y b) la de conjugar estos proyectos con el entorno social y las exigencias del bien común que ha de ser, también, definido por la ciudadanía.

El artículo está organizado en tres apartados. En el primero, explicamos el proyecto pedagógico en el cual se inserta esta experiencia y la metodología utilizada que, como señalamos, está inspirada en el modelo teórico de las éticas dialógicoprocedimentales. En el segundo, se exponen los resultados de una evaluación hecha de esta experiencia, que buscaba levantar datos sobre los recursos utilizados por nuestros/as estudiantes, así como las dificultades y limitaciones identificadas en el proceso. En la tercera y final, ofrecemos una discusión de nuestros resultados, así como algunas reflexiones que nos parecen de importancia en relación con la formación ciudadana de nuestros jóvenes.

\section{2. ÉTICA Y COMPETENCIAS: HACIA UNA METODOLOGÍA DE TRABAJO}

Si bien distintas encuestas sugieren que las personas jóvenes tienden a ser más tolerantes que el conjunto de la población (ICSO-UDP, 2010), la tolerancia -entendida como la admisión del derecho de ciertos grupos percibidos como minoritarios a desarrollar sus propios planes de vida- no necesariamente da cuenta del interés o la capacidad de construir un marco de acción común en el que todos los miembros de la sociedad tengan la oportunidad de llevar vidas que tengan razones para valorar, al decir de Sen (2000), lo que sería la marca del desarrollo humano.

Con estas consideraciones - la diversidad de visiones de mundo y el acelerado cambio social- como trasfondo, el módulo Ética y Ciudadanía, del Programa de Formación Fundamental (PFF) de la Universidad de $\mathrm{Talca}^{1}$, se propuso fortalecer la capacidad de diálogo cívico y de tomar decisiones en acuerdo con otros como uno de sus objetivos. Ello, como una forma de optimizar la incorporación de los egresados a un mundo laboral cambiante, pero de un modo que en forma paralela potenciara "una educación para la ciudadanía responsable, que incluye la necesidad de desarrollarse como persona y ser capaz de afrontar responsabilidades sociales" (González \& Wagenaar, 2003: 76). Desde esta perspectiva, se puede comprender que el módulo Ética y Ciudadanía ${ }^{2}$ buscara contribuir a la formación de profesionales capaces de reflexionar sistemáticamente sobre sus opcionales valóricas personales y profesionales, proporcionándoles un fundamento

$1 \quad$ El PFF fue creado mediante la Resolución 082 (promulga acuerdo de la Junta Directiva que aprueba el perfil genérico y la estructura curricular del grado de Licenciado y Título Profesional) para el desarrollo de competencias socioemocionales en los estudiantes de pregrado, en el marco de la transformación curricular iniciada por esa casa de estudios con financiamiento del proyecto MECESUP TAL0101.

2 Entre 2006 y 2010 el módulo tuvo el nombre de Ética, Valoración y Sociedad, pasando a llamarse Ética y Ciudadanía a partir de 2011, y se imparte a todas las carreras de la universidad. 
ético en su actuar como ciudadanos/as. Las competencias comprometidas en el módulo de Ética y Ciudadanía son las siguientes:

1. Identifica los fundamentos éticos de la limitación de su propia libertad en relación con las libertades de otras personas en su comunidad.

2. Identifica los fundamentos éticos de la existencia de cursos de acción diversos o contradictorios, siempre que estos se ajusten a mínimos éticos previamente consensuados por la colectividad (derechos/deberes).

3. Fundamenta sus opciones valóricas sobre la base de una reflexión basada en principios universalizables para la construcción de acuerdos democráticos.

4. Delibera, en conjunto con otros, fundamentando su postura personal para la construcción de acuerdos ${ }^{3}$.

Esta última competencia - la deliberación- es el foco central de este artículo. En concordancia con el modelo por competencias adoptado por la universidad, que exige el desarrollo de habilidades y capacidades transferibles desde el aula hacia situaciones profesionales, la metodología utilizada en el curso privilegió poner a los estudiantes en situaciones de conflicto, de controversias o dilemas que les ayudaran a transitar desde una posición entendida como producto de costumbres recibidas o aprendidas -sin que medie una habilidad analítica en su juicio o valoración- hacia una que sea el resultado de reflexiones y decisiones personales informadas y fundadas. Nos propusimos crear espacios de discusión que permitieran hacer la distinción entre las diversas ofertas de vida buena que existen en una sociedad plural y la existencia de mínimos éticos que hay que valorar y practicar, pensando en el bien propio y el de toda la comunidad.

En este sentido la educación y especialmente, la llamada educación moral tiene que ver con la formación de una personalidad consciente, capaz de ejercer su libertad pero también de respetar y ser consciente de sus deberes, con habilidad para enfrentarse a la indeterminación humana y "moverse equilibradamente en los planos personales y colectivo para asegurar la creación de formas de vidas viables, personalmente deseables y colectivamente justas y libres" (Puig, 1996: 18). Así, la formación en valores éticos y para la ciudadanía en el mundo universitario no se agota en un conjunto de saberes más o menos bien asimilados, sino que debe suponer un aprendizaje práctico, vivido en la cotidianidad de la vida del estudiante que combine en la construcción de su forma de ser, pensar y convivir, criterios de felicidad personal con criterios de solidaridad y cuidado hacia los demás. Como señala Miquel Martínez: "Educar en valores es promover actitudes y disposiciones en la persona favorables para la transformación de su entorno en un medio más equitativo, democrático y digno para todas y cada una de las personas que en él conviven" (Cit. en Bara, 2004: 15-16). Para esta tarea, es necesario cultivar la capacidad de explicitar las razones detrás de nuestras decisiones en el plano valórico y de ser capaces de comprender-aunque no necesariamente de compartir- las motivaciones y jerarquías de valores de otras personas, que en tanto seres humanos tienen derecho a plantear sus concepciones

3 Syllabus de Ética y Ciudadanía, Programa de Formación Fundamental, Universidad de Talca. 
sobre lo justo y lo bueno en un pie de igualdad (Rawls, 1993). Esta capacidad es sustantiva a la hora de pensar en fortalecer la autonomía moral y los valores cívicos que sustentan la vida democrática, aspecto que nos propusimos desarrollar, como explicamos en la sección siguiente.

\section{LA EXPERIENCIA DEL MÉTODO DELIBERATIVO}

Como observan Ryan \& Bisson (2011), la enseñanza de la ética se refiere a la capacidad de hacer que los/as estudiantes sean conscientes de sus propias opciones personales y jerarquía de valores, y cómo estas pueden entrar en conflicto con normas existentes. A ello, podemos añadir la existencia de desafíos nuevos, no anticipados por la educación recibida: las transformaciones tecnológicas y el avance del conocimiento en un mundo en constante cambio propician el surgimiento de controversias éticas o dilemas que serán la norma más que la excepción. En este contexto, es imprescindible fortalecer la capacidad del estudiantado para tomar decisiones -razonables y prudentes- pensando que pertenecen a una generación que se enfrentará con problemas inéditos en el mundo del trabajo y las consecuencias de sus decisiones no solo afectarán a sus coetáneos, sino que también a generaciones futuras.

Este objetivo es particularmente compatible con el modelo basado en competencias, concepto que hemos entendido en una doble perspectiva ${ }^{4}$. En primer lugar como una combinación entre destrezas, habilidades y conocimientos necesarios para desempeñar una tarea específica (Le Boterf, 2001). Así, la competencia incluye tanto medios como fines, donde los conocimientos, habilidades y destrezas se consideran como medios que permiten alcanzar el fin: desempeñar efectivamente una tarea o actividad cumpliendo con los estándares requeridos en un determinado oficio. Por este motivo, toma relevancia el concepto de aprendizaje basado en el desempeño que aspira a ser capaz de documentar los logros que han obtenido los/as estudiantes en la adquisición de una o varias competencias (González et al., 2009).

Así, y siguiendo los supuestos del modelo de enseñanza-aprendizaje basado en competencias, optamos por metodologías activas y participativas, que permitieran a los/as estudiantes tomar conciencia de sus prejuicios y tener la oportunidad de elaborar juicios fundados, sometidos a diálogo y discusión colectiva, así como desarrollar la capacidad de ponerse en el lugar del otro en el sentido de hacer esfuerzos por entender, aceptar o refutar argumentos diferentes. En suma, considerar a los otros como interlocutores válidos. Ello, junto con el esfuerzo para buscar acuerdos y validar principios que regulen el comportamiento de todos los participantes de una comunidad educativa.

Para lograr este objetivo, se estableció una secuencia o recorrido de aprendizaje que iba desde comprender conceptos clave de la ética contemporánea, hasta aplicarlos en la deliberación con otros. La secuencia, y sus correspondientes instrumentos de evaluación, se resumen en la Tabla 1:

4 Estas definiciones conceptuales y las metodologías de aprendizaje se encuentran expuestas en Gómez y Royo (2012). 
Tabla 1. Competencias e instrumentos de evaluación - Módulo Ética y Ciudadanía

\begin{tabular}{|l|l|l|}
\hline Competencia principal a evaluar & Componente principal & $\begin{array}{c}\text { Instrumento de } \\
\text { evaluación }\end{array}$ \\
\hline $\begin{array}{l}\text { Identifica los fundamentos éticos de la limita- } \\
\text { ción de su propia libertad en relación con las } \\
\text { libertades de otras personas en su comunidad. }\end{array}$ & $\begin{array}{l}\text { Identifica los fundamentos éticos de la existencia } \\
\text { de cursos de acción diversos o contradictorios, } \\
\text { siempre que estos se ajusten a mínimos éticos } \\
\text { previamente consensuados por la colectividad } \\
\text { (derechos/deberes). }\end{array}$ & Cognitivo \\
\hline $\begin{array}{l}\text { Fundamenta sus opciones valóricas sobre la } \\
\text { base de una reflexión basada en principios uni- } \\
\text { versalizables para la construcción de acuerdos } \\
\text { democráticos. }\end{array}$ & Cognitivo-procedimental & $\begin{array}{l}\text { Análisisis de casos } \\
\text { (individual) }\end{array}$ \\
\hline $\begin{array}{l}\text { Delibera, en conjunto con otros, fundamentan- } \\
\text { do su postura personal para la construcción de } \\
\text { acuerdos. }\end{array}$ & $\begin{array}{l}\text { Cognitivo-procedimental- } \\
\text { actitudinal }\end{array}$ & $\begin{array}{l}\text { Método deliberativo } \\
\text { (grupal) }\end{array}$ \\
\hline
\end{tabular}

El cuadro anterior debe ser leído considerando que el modelo basado en competencias no considera que cada etapa del recorrido de aprendizaje desemboca en competencias "finales": a diferencia de los contenidos puramente cognitivos, las competencias -y particularmente las del tipo socioemocional, como lo es la reflexión ética- siguen desarrollándose a lo largo de toda la vida. Se trata, no obstante, de fijar niveles de logro que puedan garantizar que los/as estudiantes cuentan con las herramientas básicas para seguir cultivando sus capacidades de autodescubrimiento moral y de diálogo cívico. Estimamos necesario aclarar que creemos que trabajar con estudiantes universitarios implica asumir que se trata de personas adultas que ya traen consigo una visión de mundo y una jerarquía de valores que constituye el punto de partida de un curso de esta naturaleza. En la medida en que se masifica la educación superior chilena, resulta razonable suponer que cada vez nos encontraremos con estudiantes cuya experiencia y opciones en el campo de las actitudes, disposiciones y valores será extremadamente heterogénea, lo que nos planteaba el desafío de enfrentar esa diversidad como parte del proyecto educativo. Ello es especialmente importante cuando se realiza en el contexto de una institución de educación superior estatal y no confesional, como es la experiencia en comento.

Desde esa perspectiva, el módulo se planteó como objetivo entregar al estudiantado las herramientas necesarias para comprender las sociedades modernas como espacios donde prima la pluralidad de jerarquías de valores y visiones de mundo. Este contexto plantea, por el hecho de la diversidad, la necesidad de establecer principios universalizables como base para la toma de decisiones comunes; principios que se derivan de un acuerdo democrático alcanzado por la vía de la deliberación. Estos principios, conceptualizados por algunos autores como mínimos morales (Rawls, 1993; Lipovestky, 2003; Cortina, 2009) incluyen aquellos acuerdos sociales que se consideran cruciales para la convivencia democrática en sociedades pluralistas donde no existe una ética sustantiva compartida por toda la población. Con ello, se enfatiza la importancia de las ideas de diálogo y 
tolerancia y el respeto a concepciones distintas de vida buena, siempre que se respeten ciertos parámetros básicos consensuados como tales. En el decir de Lipovetsky: "Para que las sociedades liberales se mantengan, no es necesario que todos compartamos los mismos valores, basta con que se acepten los valores mínimos de la democracia y con que domine el ethos práctico de la tolerancia” (Lipovestky, 2003: 51).

Esta competencia, formulada como la capacidad de deliberar con otros para alcanzar acuerdos, es la de mayor complejidad y, para lograrla, el trabajo en sala y los instrumentos de evaluación estuvieron inspirados en el procedimiento de las éticas dialógicas, en la línea propuesta por Habermas (1987), Apel (1991) y Behabib (1990), entre otros autores. Para ello, se organizó al curso en sesiones de deliberación y juego de roles en torno a problemáticas para las que debía proponerse una solución que cumpliera con los siguientes criterios: a) debían fundamentarse los conceptos y marcos analíticos revisados en el módulo; b) los participantes debían ponderar las soluciones propuestas tanto desde el punto de vista de su ajuste a principios éticos como de las consecuencias que implicaban para cada uno de los actores involucrados en el problema, y c) debían cumplir el criterio de la regla de validez pública: la decisión tenía que ser aceptada por todos los actores potencial o actualmente afectados por la decisión (Habermas, 1996). Así, a diferencia del debate como instrumento pedagógico, el método deliberativo tiene como función principal ejercitar "la búsqueda cooperativa de la verdad", como la llamara Habermas (1987 y 1996). Por ello, no se trata de ganar con los mejores argumentos, sino de permitir que las mejores razones puedan ser exploradas y elaboradas cooperativamente. Como observa Burbules, el diálogo, en esta vertiente:

Supone un compromiso con el proceso mismo de intercambio comunicativo, una disposición a 'llevar las cosas hasta el fin' para llegar a entendimientos o acuerdos significativos entre los participantes. Aparte de eso, muestra una actitud de reciprocidad entre los participantes, un interés, respeto y cuidado de cada uno ante los demás, aun ante los desacuerdos (1999: 31).

Si bien los valores no pueden ser observados directamente, los comportamientos y las decisiones asociadas a ellos sí pueden serlo (Van Deth \& Scarbrough, 2004). Por este motivo, la evaluación final del módulo estuvo estructurada como un caso abierto, que los estudiantes debían investigar y ante el cual tendrían que posicionarse y decidir. Para ello, se dividió al curso en grupos de hasta 12 personas, cada uno de los cuales debía investigar un mismo dilema. Este grupo mayor fue a su vez dividido en subgrupos de tres estudiantes cada uno, que debía investigar el caso desde la perspectiva de uno de los actores involucrados de acuerdo con una guía de trabajo entregada por las docentes. Los actores sociales fueron asignados por sorteo. Así, en cada dilema había cuatro subgrupos investigando la misma problemática, pero desde la perspectiva de actores sociales diferentes. Por ejemplo, ante un dilema ético relacionado con el medioambiente e industria, habría un subgrupo investigando la misma situación desde la perspectiva de los ambientalistas, otro de los ciudadanos afectados, otro de las autoridades responsables y otro de los representantes de las industrias potencialmente contaminantes. Con ello, se esperaba que la investigación previa a la deliberación proporcionara al estudiantado una visión del problema con una perspectiva particular: la tarea de cada subgrupo era reconstruir el punto de vista del actor a quien representaban, para luego examinar críticamente los planteamientos investigados en términos de su ajuste a los principios examinados en el curso. En este primer ejercicio de reflexión ética, cada subgrupo debía reportar en qué 
medida los planteamientos del actor al cual debían representar eran compatibles con principios universalizables y, sobre la base de dicho análisis, proponer una solución.

Luego, en una sesión en sala, los subgrupos que representaban a cada actor social fueron reordenados para constituir comités de deliberación compuestos por un representante de cada actor social. Esto es, el Comité estaba compuesto por representantes de cada subgrupo: todos habían investigado el mismo dilema, pero desde la perspectiva de diferentes actores. El desafío, en esta etapa del trabajo, era alcanzar una solución tan cercana al consenso como fuera posible, examinando cada uno de los argumentos y soluciones propuestos a la luz de su aplicabilidad y a la regla de validez pública, nuevamente según una guía entregada por las docentes. En ella, los integrantes del Comité debían registrar sus razones a favor y en contra de cada solución, su aplicabilidad (¿es una solución viable?) y su validez pública (¿aceptan esta solución todos los actores? ¿Por qué?). En esta guía se incluyó además un ítem en el que se pedía al estudiantado que señalara las principales dificultades encontradas en el proceso de deliberación. Es importante hacer explícito que los criterios de evaluación para cada etapa estaban basados en el cumplimiento del procedimiento señalado y la fundamentación de la decisión tomada, y no en los valores priorizados por cada grupo para avalar la solución propuesta.

En cada una de las evaluaciones del módulo se pidió a los/as estudiantes que completaran una breve encuesta de percepción, que buscó registrar tanto los recursos utilizados para cada uno de los productos (evaluaciones) como sus impresiones del proceso. Ello, con el fin de analizar dificultades y posibilidades de fortalecer en el estudiantado los componentes cognitivos, procedimentales y actitudinales que se requiere movilizar para el logro de las competencias comprometidas. Los resultados se presentan en la sección siguiente.

\section{RESULTADOS: EVALUANDO LA EXPERIENCIA}

El grupo de estudiantes que participó en este módulo $(\mathrm{N}=42)$ y cuyos resultados presentamos a continuación pertenece a la Carrera de Agronomía de la Universidad de Talca, en su quinto semestre de carrera (primer semestre de 2013). La experiencia nos enseña que los/as estudiantes de carreras científicas, con frecuencia acostumbrados a las certezas de las ciencias exactas, presentan al comienzo una cierta resistencia frente a discursos que, a su juicio, no cumplen con los criterios de universalidad, replicabilidad y comunicabilidad propias del razonamiento científico. Por tanto, el primer desafío fue trabajar con ellos la racionalidad práctica, propia de la ética, que habilita para la resolución del cada caso y en donde la virtud de la prudencia juega un rol fundamental. En este ámbito del saber los contextos concretos son de particular importancia, ya que se trata de orientarnos en un aspecto singular de la vida humana: perfeccionar la praxis, es decir, nuestra capacidad de elegir y decidir no solo antes cuestiones sustantivas de la vida personal, sino que también en aquellas relacionadas con el ámbito de lo público.

En este escenario, una de las primeras constataciones de la experiencia del método deliberativo fue que para los/as estudiantes alcanzar acuerdos resultó una tarea compleja. La encuesta de percepción aplicada tras cada evaluación preguntó, utilizando escalas tipo Likert, por el grado de dificultad percibido en cada producto. Como punto de comparación para este desafío en particular hemos tomado las otras dos evaluaciones del módulo: 
Tabla 2. Dificultad percibida por evaluación - Porcentaje que marcó la opción $(\mathrm{N}=42)$

\begin{tabular}{|l|c|c|c|}
\hline \multirow{2}{*}{ Grado de dificultad percibido } & \multicolumn{3}{|c|}{ Producto } \\
\cline { 2 - 4 } & Análisis de textos & Análisis de caso & Método deliberativo \\
\hline Fácil & 2,5 & 4,8 & 2,4 \\
\hline Dificultad moderada & 71,8 & 73,8 & 42,9 \\
\hline Difícil & 23,1 & 16,6 & 42,9 \\
\hline Muy difícil & 2,6 & 4,8 & 11,9 \\
\hline Total & 100 & 100 & 100 \\
\hline
\end{tabular}

Nota: originalmente la escala consideraba también la opción "Muy fácil", que no fue seleccionada en ninguno de los productos.

Como puede observarse, los/as estudiantes percibieron este último producto como el más arduo: 55\% de ellos/as lo calificó de "difícil" o "muy difícil", frente a 49\% del análisis de textos y $21,4 \%$ del análisis de casos. Una clave para entender el origen de estas dificultades la encontramos en los datos cualitativos obtenidos a partir del reporte hecho por los/as estudiantes en la guía de trabajo proporcionada para el método deliberativo que, como señalamos, incluyó un ítem en el que se pedía al estudiantado que indicara las principales dificultades encontradas en el proceso de deliberación. Los resultados de la categorización de esta pregunta abierta se presentan a continuación:

Tabla 3. Principales dificultades del proceso de deliberación

(puede indicarse más de una razón) - Porcentaje que marcó la opción ( $\mathrm{N}=14)^{*}$

\begin{tabular}{|l|c|}
\hline \multicolumn{1}{|c|}{ Categoría de razones } & Porcentaje \\
\hline Presentar argumentos/evidencias (cifras, datos, noticias) para defender la propia postura & $17 \%$ \\
\hline Escuchar puntos de vista con los que no se está de acuerdo & $32 \%$ \\
\hline Representar un actor cuyo punto de vista no se comparte & $53 \%$ \\
\hline Persuadir a miembros particularmente "duros" (in vivo) del grupo & $37 \%$ \\
\hline Lograr que el grupo tratara de llegar a un acuerdo que dejara satisfechos(as) a todos(as) & $74 \%$ \\
\hline
\end{tabular}

(*) Por tratarse de un trabajo grupal, cada guía se ha contabilizado como un caso.

De acuerdo con lo señalado por el alumnado, las mayores dificultades en la deliberación radicaron en tener que "ponerse en los zapatos" de un actor cuya perspectiva no se compartía, persuadir a quienes se mostraban particularmente reticentes a dejarse persuadir y lograr un acuerdo por consenso. Este último fue, comprensiblemente, el mayor desafío encontrado. A diferencia de otra metodología dialógica -el debate- el método deliberativo introduce matices en la multiplicidad de posturas/actores (y no solo a favor y en contra) y en la exigencia de que la solución sea aceptable por todos quienes resultan afectados por ella. Implica, así, un doble esfuerzo: buscar y fundamentar las razones por las que un determinado actor social presenta una posición frente a un dilema ético, y utilizar la racionalidad comunicativa (diálogo) como único instrumento que les permite alcanzar acuerdos producto 
de la deliberación y posterior validación de ciertos principios éticos en el contexto de la regla de validez pública. Por ello, los/as jóvenes deben movilizar una cantidad importante de recursos académicos y personales, como se muestra en el cuadro siguiente:

Tabla 4. En una escala del 1 al 5 donde 1 sea "Muy poco importante" y 5

"Muy importante", ¿qué recursos personales consideras que debiste utilizar para esta evaluación? Solo suma porcentajes "bastante importante" y "muy importante" $(\mathrm{N}=42)$

\begin{tabular}{|l|c|c|c|}
\hline \multirow{2}{*}{\multicolumn{1}{|c|}{ Recurso }} & \multicolumn{3}{c|}{ Tipo de evaluación } \\
\cline { 2 - 5 } & $\begin{array}{c}\text { Análisis } \\
\text { de textos }\end{array}$ & $\begin{array}{c}\text { Análisis } \\
\text { de caso }\end{array}$ & $\begin{array}{c}\text { Método } \\
\text { deliberativo }\end{array}$ \\
\hline La capacidad de memorizar lo leído/visto en clases & 20,5 & 14,3 & 33,3 \\
\hline La capacidad de comprender lo leído/visto en clases & 87,1 & 64,3 & 73,8 \\
\hline Conocimiento previo sobre los temas tratados & 56,4 & 33,3 & 59,5 \\
\hline Discusiones sostenidas en clases & 59,0 & 71,4 & 71,4 \\
\hline Discusiones sostenidas fuera de clases (con tus amigos, familia, pareja) & 28,2 & 38,1 & 47,6 \\
\hline
\end{tabular}

Como revela la Tabla 4, el porcentaje de estudiantes que reportó haber utilizado los recursos listados arriba fue mayor para el método deliberativo en todos los ítems, excepto la capacidad de comprender lo visto en clases -que, aun así, llegó a 74\%-. Esto último, posiblemente, se relacione con la naturaleza de la deliberación propiamente tal que, en su parte práctica, involucraba menos la movilización de conceptos (aunque el de mínimos y máximos morales fue frecuentemente escuchado en las argumentaciones) y más la capacidad de explicar y fundamentar. Eso explicaría, al menos parcialmente, la importancia creciente adquirida por la experiencia de discutir dentro y fuera de clases.

La experiencia personal, como muestra la Tabla 5, también fue un insumo importante para todas las evaluaciones, pero particularmente para el método deliberativo:

Tabla 5. En una escala de 1 a 5, siendo 1 "Muy poco importante" y 5 "Muy importante", ¿qué grado de importancia le asignarías a tu experiencia (personal) para enfrentar exitosamente esta evaluación? Porcentaje que marcó la opción $(\mathrm{N}=42)$

\begin{tabular}{|l|c|c|c|}
\hline \multirow{2}{*}{$\begin{array}{c}\text { Grado de importancia } \\
\text { percibido }\end{array}$} & \multicolumn{3}{c|}{ Tipo de evaluación } \\
\cline { 2 - 4 } & Análisis de textos & Análisis de caso & Método deliberativo \\
\hline Poco importante & 2,6 & 0,0 & 2,4 \\
\hline Moderadamente importante & 25,6 & 26,2 & 21,4 \\
\hline Bastante importante & 48,7 & 61,9 & 52,4 \\
\hline Muy importante & 23,1 & 11,9 & 23,8 \\
\hline Total & 100 & 100 & 100 \\
\hline
\end{tabular}

Nota: originalmente la escala consideraba también la opción "Muy poco importante", que no fue seleccionada en ninguna de las evaluaciones. 
Ya en el primer producto, $72 \%$ de los/as estudiantes reportó haber movilizado su experiencia personal (y por tanto, su propia vivencia y jerarquía de valores) como elemento "bastante" o "muy importante" para la evaluación. Este porcentaje subió a 74\% en el análisis de caso, y a 76,2\% en el método deliberativo.

Otro dato que nos importaba relevar para evaluar esta experiencia era la posibilidad de aplicar las habilidades desarrolladas a otros contextos de su vida, un eje fundamental del modelo por competencias (Le Boterf, 2001). Nuevamente, hemos tomado las otras dos evaluaciones como punto de comparación, como se muestra en la Tabla 6 .

Tabla 6. En una escala del 1 al 5 donde 1 sea "Muy poco importante" y 5 "Muy importante", ¿en qué contexto(s) consideras que podrías aplicar lo aprendido hasta aquí en el módulo?

Solo suma porcentajes "bastante importante" y "muy importante" $(\mathrm{N}=42)$

\begin{tabular}{|l|c|c|c|}
\hline \multirow{2}{*}{ Contexto de aplicación } & \multicolumn{3}{c|}{ Evaluación } \\
\cline { 2 - 4 } & $\begin{array}{c}\text { Análisis } \\
\text { de textos }\end{array}$ & $\begin{array}{c}\text { Análisis } \\
\text { de caso }\end{array}$ & $\begin{array}{c}\text { Método } \\
\text { deliberativo }\end{array}$ \\
\hline Solo en este curso & 12,8 & 16,7 & 23,8 \\
\hline En otros cursos de la carrera que estudio & 51,3 & 54,7 & 61,9 \\
\hline $\begin{array}{l}\text { En mis relaciones cotidianas con personas cercanas: familia, } \\
\text { amigos, pareja }\end{array}$ & 79,5 & 78,6 & 78,6 \\
\hline $\begin{array}{l}\text { En mis relaciones cotidianas con personas en el contexto } \\
\text { universitario o laboral (colegas, profesores, administrati- } \\
\text { vos, autoridades, etcétera) }\end{array}$ & 74,4 & 88,1 & 85,7 \\
\hline En decisiones en mi vida profesional & 76,9 & 85,7 & 66,7 \\
\hline En decisiones en mi vida personal & 84,6 & 88,1 & 71,4 \\
\hline
\end{tabular}

Como muestra la Tabla 6 se reportan altos niveles de aplicabilidad en otros contextos, particularmente el círculo cercano y profesional, seguidas de las decisiones en la vida personal y profesional. En el caso de estas dos últimas, el método deliberativo muestra una baja apreciable frente a las evaluaciones anteriores. Una hipótesis sobre este resultado es que, mientras las dos primeras evaluaciones fueron diseñadas por las docentes tanto en forma como en contenido, cuidando que cubrieran tanto aspectos profesionales como de relaciones humanas que (la experiencia nos indica) resultaban interesantes para los jóvenes, en la tercera se permitió al curso escoger los temas que serían objeto de deliberación. Frente a una gran diversidad de intereses, esto implicó que no todos estuvieran de acuerdo con los temas escogidos o con el actor asignado y que, por ello, sintieran que les "tocaban" menos. Ello, por supuesto, también nos reenvía a una dificultad o limitación importante de esta experiencia: que el estudiantado consiguiera distinguir las cuestiones sustantivas planteadas por los dilemas éticos escogidos de los aspectos procedimentales. Y es que estos últimos son lo que pueden transferirse más fácilmente a otros contextos.

De la misma manera, lo anterior nos señala que desarrollar la capacidad de "ponerse en los zapatos del otro" es una tarea compleja y una dificultad recurrente en el desafío de fortalecer las capacidades dialógicas. Creemos que esto se relaciona, por una parte, 
con la percepción de que la ética "se aprende en casa" y que, una vez alcanzada la edad adulta, la jerarquía de valores se mantendrá inalterada por la experiencia -particularmente, por la experiencia de otros-. Por ello, mientras se vislumbra la posibilidad de transferir las capacidades de autodescubrimiento ético cultivadas en las dos primeras evaluaciones (ambas individuales ${ }^{5}$ ), el ejercicio de persuasión y búsqueda cooperativa de soluciones plantea el desafío concreto de la convivencia con otros. Como señaláramos al inicio de este artículo, en un contexto donde los grandes sistemas de sentido pierden importancia y los/as jóvenes muestran posturas de ambigüedad y desconfianza frente al proyecto colectivo, este es un desafío particularmente urgente.

\section{DISCUSIÓN Y CONCLUSIONES}

Los datos de la VII Encuesta Nacional de la Juventud con los que iniciamos este artículo también muestran que, a nivel país, 59\% de los jóvenes declara no conversar sobre política con sus familias, pares, amigos u otras personas. En la VII región, este porcentaje sube a $66 \%$, con diferencias muy leves por tramos de edad. Sin embargo, al comparar por niveles de educación, encontramos que entre quienes declaran estar en la educación terciaria (universidad, instituto profesional o CFT), 51\% reporta que sí aborda temas de política con su círculo cercano, marcando una diferencia estadísticamente significativa con quienes no están en ninguna institución de educación superior ${ }^{6}$. Ello nos sugiere que este es un espacio privilegiado para el desarrollo de las competencias dialógicas en el ámbito de la ética cívica -un espacio para cultivar la habilidad de debatir los problemas comunes de una sociedad en un marco de respeto y tolerancia. En el grupo de estudiantes encuestados durante esta experiencia educativa, 14,7\% declaró no hablar de valores con su entorno cercano nunca o hacerlo muy raramente; $42,3 \%$ tocar estos temas "raramente" y $43 \%$ hacerlo frecuente o muy frecuentemente. Así, la tendencia nacional y regional se verificó también en nuestros estudiantes. El escaso hábito de discutir temas de política o valores con sus pares o cercanos -cuestiones vinculadas a la reconstrucción del proyecto colectivo en el contexto de la erosión de los grandes sistemas de sentido- parece ser una constante entre los jóvenes.

Al respecto, coincidimos con Gracia (2004) en que un aspecto valioso del tipo de enseñanza que hemos descrito es hacer posible que los/as estudiantes se apropien de la idea que -en un mundo complejo como el que vivimos- existen razones, valores y creencias que no es posible racionalizar del todo, porque pertenecen al ámbito vital de nuestros valores más profundos, pero de las que sí tenemos que ser capaces de dar razones, "a fin de exigirnos a nosotros mismos y exigir a los demás que sus decisiones sean, no uniformes, pero sí razonables y prudentes" (Gracia, 2004: 37). Por ello, se hace necesario crear espacios en los que nuestros jóvenes puedan construir una comprensión más reflexiva de sí mismos -una comprensión que, por definición, debe estar basada en las jerarquías de valores que ellos/as portan (Burbules, 1999: 34-35). Desde el punto de

\footnotetext{
5 Aquí es importante señalar que, si bien la evaluación fue individual, gran parte del trabajo en sala previo fue hecho en parejas o grupos de tres personas.

$6 \quad$ Agradecemos al INJUV por habernos proporcionado la base de datos de la VII Encuesta Nacional de la Juventud, lo que hizo posible hacer algunos análisis específicos a la VII Región.
} 
vista educacional, se hace necesario buscar una relación equilibrada y eficaz que armonice los saberes expertos propios de una disciplina con competencias de tipo socioemocional que permitan al estudiantado instalarse en el mundo con mayores niveles de confianza, flexibilidad, compromiso ético, tolerancia y autonomía personal.

Lo anterior contribuye con el fortalecimiento de la autonomía moral y la necesidad imperiosa de contar con argumentos que permitan a nuestros estudiantes diseñar su proyecto de vida personal y profesional, teniendo a la vista no solo su propia visión de felicidad o alternativa de vida buena, sino que también pensar de qué manera se busca el progreso moral de una sociedad. La educación en este plano se relaciona con la necesidad de que los/as estudiantes cultiven su capacidad de autodescubrimiento ético y adquieran la cultura moral necesaria para enfrentarse autónoma y dialógicamente a aquellas situaciones que suponen un conflicto de valores o controversia moral, situaciones que, según la sociología de la modernidad, serán cada vez más frecuentes en los años venideros. Ello, de forma que le sea posible vivir de un modo justo, solidario y feliz, como plantea Puig (Cit. en Martínez, 2007), sin que sus proyectos personales colisionen o entren en conflicto con los mínimos éticos deseables en una democracia: justicia, igualdad y solidaridad.

Como observa Aravena (2009), las personas jóvenes se encuentran más expuestas y abiertas a los cambios societales, por lo que este segmento es una "ventana" privilegiada para observar las tendencias normativas de cada sociedad y las tensiones que no han podido ser resueltas (Cit. en INJUV, 2012: 102). Desde este punto de vista, el que no se hable -o se hable poco- de valores nos plantea el desafío de fortalecer las capacidades de diálogo cívico. El diálogo ayuda a tender puentes, a descubrir puntos comunes entre el proyecto personal y el ethos colectivo, propiciando la reflexión sobre lo que, como sociedad, estamos dispuestos a aceptar como parte de nuestra identidad moral, personal y colectiva. Esta es una tarea que tiene dificultades no menores para docentes y estudiantes, y que -como muestra nuestra experiencia- no siempre será completada con éxito. En el método deliberativo, los/as estudiantes debieron movilizar más recursos que en las otras evaluaciones, pero también los percibieron como menos susceptibles de ser transferidos a otros contextos de aplicación. Y es que nos movemos en un ámbito del saber -la razón práctica- cuyas verdades son contingentes porque son producto de un acuerdo colectivo que está sujeto a cambios en virtud de las condiciones sociales, culturales y tecnológicas de la sociedad. Esto es también, por supuesto, lo que abre la posibilidad de aprendizaje moral y ético de las sociedades humanas, como planteara Habermas (1987).

Considerando la historia reciente de América Latina, creemos que el fortalecimiento de las competencias dialógicas es esencial para la protección de nuestras frágiles democracias, particularmente frente a los procesos de destradicionalización y reflexividad (Giddens, 2000). Pues si bien dichos procesos abren la posibilidad de mayor individuación y libertad personal, pueden tender a la fragmentación si no nos ocupamos de reconstruir, constantemente, los acuerdos colectivos que son fundamentales para (con)vivir.

\section{REFERENCIAS BIBLIOGRÁFICAS}

Apel, K. (1991). Teoría de la verdad y ética del discurso. Barcelona: Editorial Paidós Ibérica.

Aravena, A. (2009). Discriminación e intolerancia. En INJUV, Sexta encuesta nacional de la juventud (pp. 311-323). Santiago: Instituto Nacional de la Juventud. 
Bara, F. (2004). Excelentes profesionales y comprometidos ciudadanos. Bilbao: Editorial Desclée.

Benhabib, S. (1990). In the shadow of Aristotle and Hegel: Communicative ethics and current controversies in practical philosophies. In M. Kelly (Ed.), Hermeneutics and critical theory in politics (pp. 1-31). Cambridge: MIT Press.

Burbules, N. (1999). El diálogo en la enseñanza. Teoría y práctica. Buenos Aires: Amorrortu.

Cortina, A. (2009). Ciudadanos del mundo: Hacia una teoría de la ciudadanía (3 ${ }^{\text {era }}$ Ed.). Madrid: Alianza.

Giddens, A. (2000). Un mundo desbocado. Los efectos de la globalización en nuestras vidas. Madrid: Taurus.

Gómez, V. y Royo, P. (2012). Ética cívica: Aprender de la experiencia vivida. Revista Calidad de la Educación, (13), 205-221.

González, E., Herrera, R. y Zurita, R. (2009). Formación basada en competencias: Desafíos y oportunidades. En V.V.A.A, Diseño curricular basado en competencias y aseguramiento de la calidad en educación superior (pp. 16-28). Santiago: CINDA.

González, J. \& Wagenaar, R. (Eds.). (2003). Tuning educational structures in Europe. Final report. Phase one. Bilbao - Gröningen: University of Deusto - University of Gröningen.

Gracia, D. (2004). La enseñanza de la bioética. En F. Lolas (Ed.), Diálogo y cooperación en salud. Diez años de bioética en la OPS (pp. 23-38). Santiago: OPS.

Habermas, J. (1996). Between facts and norms. Towards a discursive theory of democracy. Cambridge: MIT Press. (1987). La teoría de la acción comunicativa (Vol. 2). Madrid: Taurus.

ICSO-UDP (2010). Encuesta nacional UDP 2010. Recuperado el 22 de diciembre de 2013 desde http://www.encuesta.udp.cl/encuestas-anteriores/encuesta-2010/

INJUV (2012). Séptima encuesta nacional de la juventud. Santiago: Instituto Nacional de la Juventud.

Le Boterf, G. (2001). Ingeniería de las competencias. París: Editorial Gestión 2000.

Lipovetsky, G. (2003). Metamorfosis de la cultura liberal. Barcelona: Anagrama.

Martínez, P. (2007). Virtud y racionalidad práctica en la educación moral. Veritas, vol. 2 (17), 313-329.

Puig, J. (1996). La construcción de la personalidad moral. Barcelona: Editorial Paidós Ibérica.

PNUD (2012). Bienestar subjetivo: El desafío de repensar el desarrollo. Santiago: Programa de las Naciones Unidas para el Desarrollo.

Rawls, J. (1993). Political liberalism. New York: Columbia University Press.

Ryan, T. \& Bisson, J. (2011). Can ethics be taught? International Journal of Business and Social Science, vol. 2 (12), 44-52.

Sen, A. (2000). Desarrollo y libertad. Buenos Aires: Planeta.

Van Deth, J. \& Scarbrough, E. (2004). The concept of values. In J. Van Deth \& E. Scarbrough (Eds.), The impact of values (pp. 21-47). Oxford: Oxford University Press. 